\title{
Comment on "Relationship between non-alcoholic fatty liver disease and benign prostatic hyperplasia/lower urinary tract symptoms: new insights from an Italian cross-sectional study"
}

\author{
Hakkı Uzun
}

Received: 7 December 2014 / Accepted: 3 January 2015 / Published online: 13 January 2015

(C) Springer-Verlag Berlin Heidelberg 2015

Dear editor,

We congratulate the authors for this interesting article about the relationship between non-alcoholic fatty liver disease (NAFLD) and benign prostatic hyperplasia (BPH) and lower urinary tract symtpoms (LUTS). However, we believe that there are some points we would like to state.

In this study, a significant relationship was put forward with FLI (fatty liver index) and LUTS and BPH. FLI was indicated and used as the predictor of NAFLD. However, FLI is an algorithm based on body mass index, waist circumference, triglycerides and gamma-glutamyl transferase which have been extensively studied and showed to be associated with BPH and LUTS in several studies.

Furthermore, the authors reported that there is a lack of data; however, NAFLD was previously reported to be associated with BPH in men and overactive bladder in women [2]. After the initial studies about the etiopathogenetic relation of BPH and metabolic syndrome and insulin resistance by Hammarsten et al. [1], there is an increasing evidence regarding the relationship between metabolic derangement and LUTS for both men and women. However, it is well known that NAFLD is strongly associated with hepatic and adipose tissue insulin resistance, as well as reduced whole body insulin sensitivity and is considered the hepatic manifestation of metabolic syndrome. Insulin resistance and metabolic syndrome are the common risk factors for both $\mathrm{BPH}$ and NAFLD; therefore, we agree to the authors that similar common etiopathogenetic factors may have similar trophic effects on hepatic tissue and prostate.

\section{References}

1. Hammarsten J, Högstedt B (1999) Clinical, anthropometric, metabolic and insulin profile of men with fast annual growth rates of benign prostatic hyperplasia. Blood Press 8:29-36

2. Uzun H, Oğullar S, Ünal H, Zorba OÜ, Yazar S, Kalkan M (2013) Non-alcoholic fatty liver disease is associated with benign prostate hyperplasia in men and with overactive bladder in women. Scand J Urol 47(6):497-502. doi:10.3109/21681805.2013.780258

This comment refers to the article available at doi:10.1007/s00345-014-1392-4.

H. Uzun $(\bowtie)$

Department of Urology, Recep Tayyip Erdoğan University

School of Medicine, Rize, Turkey

e-mail: hakuzun@yahoo.com 